\title{
Complicaciones del trasplante renal en el Instituto de Nefrología. 2001-2005
}

\author{
Roynel Rodríguez Martínez* - Raymed Bacallao Méndez** - Francisco Gutiérrez García** \\ Dianelys Fonseca Hernández*
}

* Enfermero/a - * Médico

Instituto de Nefrología Dr. Abelardo Buch López y Hospital Docente Clínico Quirúrgico Hermanos Ameijeiras

\section{Resumen}

Introducción y Objetivos: A pesar del desarrollo del trasplante nuestros resultados se ven afectados por la aparición de complicaciones médicas y quirúrgicas entorpeciendo su evolución. Nuestro propósito fue identificar las complicaciones más frecuentes de la primera semana post-trasplante renal y su repercusión sobre la supervivencia del paciente y del injerto al mes y al año posterior al trasplante.

Métodos: estudio observacional descriptivo de cohorte retrospectiva en el Instituto de Nefrología, de los pacientes trasplantados entre los meses de enero 2001 hasta diciembre 2005. Se analizaron las variables de interés. Se empleó la técnica estadística de análisis de distribución de frecuencias. Para cada una de las categorías de las variables fueron calculadas frecuencias absolutas y relativas (porcentajes). Se empleó el test de homogeneidad para probar la hipótesis nula (Ho), de igualdad de distribución de algunas variables con un nivel de significación $\alpha=0,05$.

Resultados: En 136 pacientes se presentaron 212 complicaciones, de ellas 159 (75\%) médicas y 53 (25\%) fueron quirúrgicas. Las complicaciones médicas más frecuentes fueron: no infecciosas 110

Correspondencia:

Roynel Rodríguez Martínez

Calle 27 \# 971 e/ 6 y 8, Plaza

Ciudad de la Habana - Cuba

roynel.rdguez@infomed.sld.cu
(51,8\%); infecciosas 33 (15,5\%). Las complicaciones quirúrgicas más frecuentes fueron: vasculares $34(16 \%)$, parenquimatosas 7 (3,3\%). Solo $30(22 \%)$ pacientes no presentaron ningún tipo de complicación y en otros $30(22 \%)$ pacientes coincidieron tanto complicaciones médicas como quirúrgicas.

Conclusiones: La ocurrencia de complicaciones médicas en la primera semana post-trasplante determina una pobre supervivencia del injerto renal a corto plazo. El hecho de presentar complicaciones quirúrgicas disminuye la supervivencia del injerto renal y del paciente trasplantado a muy corto y a corto plazo.

\section{PALABRAS CLAVE:}

- INSUFICIENCIA RENAL

- COMPLICACIONES TRASPLANTE

- SUPERVIVENCIA TRASPLANTE

Kidney transplant complications in the $\mathrm{Ne}$ phrology Institute. 2001-2005

\section{Abstract}

Introduction and Objectives: Despite the development of transplants our results are affected by the appearance of medical and surgical complications that hinder their evolution. Our aim was to identify the most frequent complications in the first week after a kidney transplant and their effects on patient and graft survival one month and one year after the transplant. 
Methods: retrospective cohort descriptive observational study at the Nephrology Institute, of patients who received transplants between the months of January 2001 and December 2005. The variables of interest were analysed. The statistical technique of frequency distribution analysis was used. For each category of variables, absolute and relative frequencies (percentages) were calculated. The homogeneity test was used to prove the null hypothesis $(\mathrm{Ho})$, of equality of distribution of some variables with a significance level $\alpha=0.05$.

Results: In 136 patients a total of 212 complications were found, of which $159(75 \%)$ were medical and $53(25 \%)$ were surgical. The most frequent medical complications were: non-infectious 110 (51.8\%); infectious 33 (15.5\%). The most frequent surgical complications were: vascular 34 (16\%), parenchymatosic 7 (3.3\%). Only 30 patients (22\%) showed no type of complication and in another 30 patients ( $22 \%$ ) there were both medical and surgical complications.

Conclusions: The occurrence of medical complications in the first week after the transplant determines poor survival of the kidney graft in the short term. The fact of showing surgical complications reduces the survival of the kidney graft and of the transplanted patient in the very short and short term.

\section{KEY WORDS:}

- RENAL INSUFFICIENCY

- TRANSPLANT COMPLICATIONS

- TRANSPLANT SURVIVAL

\section{Introducción}

La Enfermedad Renal Crónica Terminal (ERCT) constituye un síndrome clínico-humoral complejo como expresión de un deterioro irreversible de la función renal con múltiples etiologías, que evoluciona habitualmente de manera lenta y progresiva, hasta llegar al estado de uremia terminal, con todas sus complicaciones ${ }^{1}$. Durante mucho tiempo diagnosticar ERCT a un paciente era sinónimo de muy mal pronóstico a mediano y a largo plazo, pues sus opciones terapéuticas eran limitadas². Por ello la sustitución del riñón enfermo por otro sano ha sido un sueño largamente acariciado por la humanidad³.

El desarrollo de los métodos sustitutivos de la función renal en sus distintas variantes - hemodiálisis, diálisis peritoneal y trasplante renal (TR) de donante vivo o cadavérico - ha servido para rescatar de la muerte a una gran cantidad de pacientes con ERCT. EI trasplante de órganos y tejidos ha experimentado un gran desarrollo en los últimos años, a principios del siglo XXI los trasplantes han pasado a ser un tratamiento habitual y en ocasiones el único disponible para determinadas enfermedades ${ }^{4}$. En la actualidad se ha demostrado que el tratamiento ideal de la ERCT es el TR 5,6, por la mejoría de calidad de vida que ofre$\mathrm{Ce}^{7}$, así como por ser más eficaz y eficiente que la diálisis $^{5,8,9,10}$. Los pacientes pueden reincorporarse a una vida laboral activa y económicamente es menos costoso a la sociedad, en comparación con los métodos dialíticos $3,8,9$.

El receptor de un TR es un paciente de gran riesgo debido a su uremia, es un candidato quirúrgico especialmente frágil, en el que el sistema inmunológico está alterado por la propia insuficiencia renal y por la posterior inmunosupresión a que será sometido. La inmunosupresión lo hace particularmente susceptible a la sepsis. Estas circunstancias unidas a la dificultad propia de esta cirugía, hacen necesaria una técnica exacta y cuidadosa, debido a las graves consecuencias, tanto de morbilidad como de mortalidad, que suponen la existencia de complicaciones clínicas y quirúrgicas $(C-Q)^{11}$.

En Cuba con el aumento de la expectativa de vida, las enfermedades crónicas no trasmisibles siguen incrementándose y con ellas el número de pacientes que evolucionarán a la ERCT, tributarios de recibir un TR. Diversos estudios se han realizado para identificar el comportamiento de algunas de las complicaciones tempranas del TR, pero en la literatura nacional no son muy frecuentes y menos aún que traten sobre su repercusión ulterior en la funcionalidad del injerto y en la vida del paciente. Por lo anterior expuesto consideramos importante identificar las características de los pacientes trasplantados en nuestra institución así como las principales complicaciones que se presentan en la primera semana posterior al trasplante 
renal y la posible repercusión de estas complicaciones sobre el paciente, y el estado del injerto al mes y al año posterior al trasplante.

\section{Material Y Método}

Se realizó un estudio observacional descriptivo de cohorte retrospectiva. El universo de estudio estuvo constituido por la totalidad de los pacientes trasplantados renales en el Instituto de Nefrología "Dr. Abelardo Buch López" (INNEF), en el período de tiempo comprendido entre enero del 2001 y diciembre del 2005.

De los archivos del Departamento de Registros Médicos de la Institución, y de los que posee el grupo básico de trabajo de Trasplante fueron seleccionadas y revisadas exhaustivamente todas las historias clínicas y pancartas de los pacientes que recibieron un trasplante renal en el período. La Pancarta constituye un documento que se utiliza en el Servicio de Trasplante del INNEF y que consiste en un formulario destinado a llevar de forma concisa y continua los aspectos más relevantes de la evolución del paciente, resumiendo su evolución clínica, medicamentos empleados, exámenes diagnósticos y complicaciones aparecidas.

Fueron registradas la ocurrencia de complicaciones médicas y quirúrgicas y el estado del injerto y del paciente, al mes y al año de realizado el trasplante. Se consideró pérdida del injerto una creatinina sérica igual a mayor que $3 \mathrm{mg} \%$ o la entrada en diálisis. Para determinar la repercusión de las complicaciones sobre el estado del injerto y el estado del paciente, se identificaron las complicaciones más graves, frecuentes y se agruparon en médicas y quirúrgicas.

Complicaciones médicas consideradas: infecciones del tracto urinario, trombosis venosa profunda, no de la víscera injertada (TVP), necrosis tubular aguda (NTA), crisis hipertensiva (HTA), convulsiones, infarto cerebral, edema agudo del pulmón (EAP), tromboembolismo pulmonar, distres respiratorio, shock (séptico e hipovolémico) arritmias, toxicidad por ciclosporina A (CyA), y los rechazos hiperagudos, acelerados y agudos.

Complicaciones quirúrgicas consideradas: hematoma perirrenal, fístula urinaria, obstrucción urinaria, trombosis arterial, trombosis venosa, hemorragia perioperatoria, dehiscencia de la herida quirúrgica y rotura renal.
Todos los datos fueron procesados de forma automatizada, se utilizó el paquete estadístico SPSS versión 13.0. De forma general se empleó la técnica estadística de análisis de distribución de frecuencias. Para cada una de las categorías de las variables fueron calculadas las frecuencias absolutas y relativas, estas últimas fueron expresadas en porcentajes. Además se empleó el test de homogeneidad, para probar la homogeneidad de la distribución de las variables "estado del injerto" y "estado del paciente" en pacientes "con" y "sin complicaciones". Para las pruebas de hipótesis realizadas se fijó un nivel de significación $\alpha=0,05$.

\section{Resultados}

Entre los meses de enero del 2001 y diciembre del 2005 fueron trasplantados un total de 136 pacientes en el INNEF. Las principales características de dichos pacientes pueden ser observadas en la tabla 1. Con relación a la edad se puede notar, que casi el $80 \%$ de los pacientes trasplantados se encuentra entre los 20 y 49 años de edad. Predomina el sexo masculino $(61,8 \%)$ y la raza blanca es la más re-

\begin{tabular}{|c|c|c|c|}
\hline Característica & Categoría & $\mathbf{N}^{0}$ & $\%$ \\
\hline \multirow{5}{*}{ Edad } & $<20$ & 10 & 7,3 \\
\hline & $20-29$ & 25 & 18,3 \\
\hline & $30-39$ & 51 & 37,5 \\
\hline & $40-49$ & 30 & 22 \\
\hline & $>50$ & 20 & 14,7 \\
\hline \multirow{2}{*}{ Sexo } & Masculino & 84 & 61,8 \\
\hline & Femenino & 52 & 38,2 \\
\hline \multirow{2}{*}{ Color de la piel } & Blanca & 94 & 69,1 \\
\hline & No blanca & 42 & 30,9 \\
\hline \multirow{2}{*}{ Tipo de donante } & Vivo & 41 & 30,1 \\
\hline & Cadavérico & 95 & 69,9 \\
\hline \multirow{2}{*}{$\begin{array}{l}\text { Número de TR } \\
\text { realizado }\end{array}$} & Primero & 124 & 91,1 \\
\hline & Segundo & 12 & 8,9 \\
\hline \multirow{10}{*}{$\begin{array}{l}\text { Causa de } \\
\text { Insuficiencia } \\
\text { Renal Crónica } \\
\text { Terminal }\end{array}$} & No filiada & 25 & 18,4 \\
\hline & Glomerulopatías & 35 & 25,7 \\
\hline & Diabetes Mellitus & 9 & 6,6 \\
\hline & Hipertensión arterial & 12 & 8,8 \\
\hline & Enf. Poliquística & 17 & 12,5 \\
\hline & Nefropatía de reflujo & 9 & 6,6 \\
\hline & Nefroangioesclerosis & 12 & 8,8 \\
\hline & Hipoplasia renal & 3 & 2,2 \\
\hline & LES & 5 & 3,7 \\
\hline & Otras & 9 & 6,6 \\
\hline
\end{tabular}

Tabla 1. Características de interés de los pacientes trasplantados 
presentada $(69,1 \%)$. La mayoría $(69,9 \%)$ de los pacientes trasplantados ha recibido órganos de donantes cadavéricos. Las principales causas de IRCT son las glomerulopatías $(25,7 \%)$ y la enfermedad renal poliquística autosómica dominante $(12,5 \%)$, $y$ en tercer lugar la nefroangioesclerosis y la hipertensión arterial, ambas con una frecuencia del 8,8 $\%$. Se hace necesario destacar que en un porcentaje no despreciable de estos pacientes $(18,4 \%)$, no se puede precisar el origen de la IRCT.

La tabla 2 muestra la frecuencia de ocurrencia de complicaciones en la primera semana posterior al

\begin{tabular}{|c|c|c|c|c|c|}
\hline \multicolumn{4}{|c|}{ Complicaciones } & $\mathbf{N}^{0}$ & $\%$ \\
\hline \multirow{22}{*}{$\begin{array}{l}\text { 造 } \\
\text { 岕 }\end{array}$} & \multirow{4}{*}{$\begin{array}{l}\text { Infecciosas } \\
33(15.5 \%)\end{array}$} & \multicolumn{2}{|c|}{ Sepsis urinaria } & 15 & 7,07 \\
\hline & & \multicolumn{2}{|c|}{ Sepsis respiratoria } & 8 & 3,77 \\
\hline & & \multicolumn{2}{|c|}{ Sepsis oral } & 4 & 1,88 \\
\hline & & \multicolumn{2}{|c|}{$\begin{array}{l}\text { Sepsis del acceso } \\
\text { vascular }\end{array}$} & 6 & 2,83 \\
\hline & \multirow{13}{*}{$\begin{array}{l}\text { No infecciosas } \\
110(51.8 \%)\end{array}$} & \multicolumn{2}{|c|}{$\begin{array}{l}\text { Trombosis venosa } \\
\text { profunda }\end{array}$} & 6 & 2,83 \\
\hline & & \multirow{2}{*}{ NTA } & Con hemodiálisis & 42 & 19,81 \\
\hline & & & Sin hemodiálisis & 6 & 2,83 \\
\hline & & \multirow{2}{*}{ HTA } & $\begin{array}{l}\text { Urgencia } \\
\text { hipertensiva }\end{array}$ & 26 & 12,26 \\
\hline & & & $\begin{array}{l}\text { Emergencia } \\
\text { hipertensiva }\end{array}$ & 7 & 3,3 \\
\hline & & \multicolumn{2}{|c|}{ Convulsiones } & 2 & 0,94 \\
\hline & & \multicolumn{2}{|c|}{ Infarto cerebral } & 1 & 0,47 \\
\hline & & \multicolumn{2}{|c|}{ Distress respiratorio } & 3 & 1,41 \\
\hline & & \multicolumn{2}{|c|}{$\begin{array}{l}\text { Tromboembolismo } \\
\text { pulmonar }\end{array}$} & 1 & 0,47 \\
\hline & & \multicolumn{2}{|c|}{ Edema agudo del pulmón } & 7 & 3,3 \\
\hline & & \multicolumn{2}{|c|}{ Shock } & 1 & 0,47 \\
\hline & & \multicolumn{2}{|c|}{ Insuficiencia cardiaca } & 1 & 0,47 \\
\hline & & \multicolumn{2}{|c|}{ Arritmias } & 7 & 3,3 \\
\hline & Medicamentosa & \multicolumn{2}{|c|}{ Toxicidad por CyA } & 5 & 2,35 \\
\hline & & \multicolumn{2}{|c|}{ Rechazo hiperagudo } & 4 & 1,88 \\
\hline & $\begin{array}{l}\text { Inmunologicas } \\
\text { (5 }\end{array}$ & \multicolumn{2}{|c|}{ Rechazo acelerado } & 2 & 0,94 \\
\hline & & \multicolumn{2}{|c|}{ Rechazo agudo } & 5 & 2,35 \\
\hline & & \multicolumn{2}{|c|}{ SUBTOTAL } & 159 & $75 \%$ \\
\hline \multirow{11}{*}{ 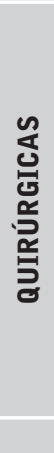 } & Urológicas & \multicolumn{2}{|c|}{ Obstrucción urinaria } & 1 & 0,47 \\
\hline & $4(1.88 \%)$ & \multicolumn{2}{|c|}{ Fístula urinaria } & 3 & 1,41 \\
\hline & & \multicolumn{2}{|c|}{ Trombosis arterial } & 10 & 4,71 \\
\hline & Vasculares & \multicolumn{2}{|c|}{ Trombosis venosa } & 3 & 1,41 \\
\hline & $34(16 \%)$ & $\begin{array}{l}\text { Hemc } \\
\text { perio }\end{array}$ & $\begin{array}{l}\text { gia } \\
\text { atoria }\end{array}$ & 21 & 10 \\
\hline & H.Q & Dehis & cia & 2 & 0,94 \\
\hline & Pararrenales & Colec & & 2 & 0,94 \\
\hline & $6(2.83 \%)$ & Hem & & 4 & 1,88 \\
\hline & Parenquimatosas & Rotur & enal & 7 & 3,3 \\
\hline & & SUBT & $A L$ & 53 & $25 \%$ \\
\hline & & TOTA & & 212 & $100 \%$ \\
\hline
\end{tabular}

Tabla 2. Complicaciones médicas y quirúrgicas de los pacientes trasplantados, en la primera semana post-TR.
TR. Se observa de manera global, que la frecuencia de complicaciones médicas ( $75 \%$ ), resulta tres veces mayor que la de complicaciones quirúrgicas (25 $\%)$. Las complicaciones médicas más frecuentes son, en orden decreciente: no infecciosas 110 (51,8 \%), representadas mayormente por la NTA y la HTA con $48(22,6 \%)$ y $35(16,5 \%)$; infecciosas $33(15,5$ $\%)$, predominando la infección del tracto urinario $15(7,07 \%)$; inmunológicas $11(5,2 \%)$, la más frecuente fue el rechazo agudo $5(2,35 \%)$ y en último lugar medicamentosas 5 (2,35\%), representada exclusivamente por la toxicidad por CyA. Las complicaciones quirúrgicas más frecuentes, también en orden decreciente, son: vasculares 34 (16\%), con mayor presentación de hemorragia perioperatoria 21 (10 $\%)$; parenquimatosas 7 (3,3\%), presentadas únicamente como rotura renal; pararrenales $6(2,83 \%)$, con mayor frecuencia de hematomas $4(1,88 \%)$; urológicas $4(1,88 \%)$, de ellas la más significativa fue la fístula urinaria $3(1,41 \%)$ y por último, dehiscencia de la herida quirúrgica $2(0,94 \%)$.

En la tabla 3 se muestra el estado del injerto al mes y al año posterior al TR según la ocurrencia o no de complicaciones médicas y quirúrgicas, encontrándose dependencia entre las variables analizadas $(p<0,05)$ en todos los casos. Los pacientes que presentaron complicaciones quirúrgicas mostraron menor funcionalidad del injerto al mes y al año post-trasplante con valores de $n(\%)$ y p: $30(73,2 \%) 0,00$ y $34(82,9$ $\%)$ 0,00 con respecto a los que no desarrollaron este tipo de complicaciones, con valores de n (\%): $14(14,7 \%), 24(25,3 \%) p=0,00$. Los pacientes que desarrollaron complicaciones médicas mostraron también menor grado de viabilidad del órgano al mes y al año post-trasplante con valores de $n(\%)$ y p: 34 $(39,5 \%) 0,02,45(52,3 \%) 0,04$ con respecto a los que no presentaron este tipo de complicaciones, con valores de $n(\%)$ y p: 10 (20\%) 0,02 y 13 (26\%) 0,04 , al mes y al año post-trasplante respectivamente.

En cuanto al estado del paciente (vivo o fallecido) al mes y al año posterior al TR, según la ocurrencia o no de complicaciones médicas y quirúrgicas (tabla 4), encontramos asociación entre la ocurrencia de complicaciones quirúrgicas y el estado del paciente al mes y al año post-TR ( $p=0,00$ en ambos casos). Hubo mayor número de fallecidos al mes y al año post-TR en el grupo de pacientes que presentaron complicaciones quirúrgicas con valores de $\mathrm{n}(\%): 7$ 


\begin{tabular}{|c|c|c|c|c|c|c|c|c|c|c|c|}
\hline \multirow{4}{*}{\multicolumn{2}{|c|}{ Complicaciones }} & \multicolumn{10}{|c|}{ Supervivencia del Injerto } \\
\hline & & \multicolumn{5}{|c|}{$1^{\text {er }}$ Mes } & \multicolumn{5}{|c|}{$1^{\text {er }}$ Año } \\
\hline & & \multicolumn{2}{|c|}{ Funcional } & \multicolumn{2}{|c|}{ No funcional } & \multirow{2}{*}{$\mathrm{p}$} & \multicolumn{2}{|c|}{ Funcional } & \multicolumn{2}{|c|}{ No funcional } & \multirow{2}{*}{$p$} \\
\hline & & $n$ & $\%$ & $n$ & $\%$ & & $n$ & $\%$ & $n$ & $\%$ & \\
\hline \multirow{2}{*}{ Médicas } & $\mathrm{Si}$ & 52 & 60,5 & 34 & 39,5 & \multirow{2}{*}{0,02} & 41 & 47,7 & 45 & 52,3 & \multirow{2}{*}{0,04} \\
\hline & No & 40 & 80 & 10 & 20 & & 37 & 74 & 13 & 26 & \\
\hline \multirow{2}{*}{ Quirúrgicas } & Si & 11 & 26,8 & 30 & 73,2 & \multirow{2}{*}{0,00} & 7 & 17,1 & 34 & 82,9 & \multirow{2}{*}{0,00} \\
\hline & No & 81 & 85,3 & 14 & 14,7 & & 71 & 74,7 & 24 & 25,3 & \\
\hline
\end{tabular}

Tabla 3. Estado del injerto al mes y al año posterior al trasplante, según ocurrencia de complicaciones médicas y quirúrgicas.

\begin{tabular}{|c|c|c|c|c|c|c|c|c|c|c|c|}
\hline \multirow{4}{*}{\multicolumn{2}{|c|}{ Complicaciones }} & \multicolumn{10}{|c|}{ Supervivencia del Paciente } \\
\hline & & \multicolumn{5}{|c|}{$1^{\text {er }}$ Mes } & \multicolumn{5}{|c|}{$\mathbf{1}^{\text {er }} \mathbf{A} \tilde{0} 0$} \\
\hline & & \multicolumn{2}{|c|}{ Vivo } & \multicolumn{2}{|c|}{ Fallecido } & \multirow{2}{*}{$\mathrm{p}$} & \multicolumn{2}{|c|}{ Vivo } & \multicolumn{2}{|c|}{ Fallecido } & \multirow{2}{*}{$\mathrm{p}$} \\
\hline & & $n$ & $\%$ & $n$ & $\%$ & & $\mathrm{n}$ & $\%$ & $n$ & $\%$ & \\
\hline \multirow{2}{*}{ Médicas } & $\mathrm{Si}$ & 79 & 91,9 & 7 & 8,1 & \multirow{2}{*}{0,25} & 77 & 89,5 & 9 & 10,5 & \multirow{2}{*}{0,32} \\
\hline & No & 49 & 98 & 1 & 2 & & 48 & 96 & 2 & 4 & \\
\hline \multirow{2}{*}{ Quirúrgicas } & $\mathrm{Si}$ & 34 & 82,9 & 7 & 17,1 & \multirow{2}{*}{0,00} & 33 & 80,5 & 8 & 19,5 & \multirow{2}{*}{0,00} \\
\hline & No & 94 & 98,9 & 1 & 1,1 & & 92 & 96,8 & 3 & 3,2 & \\
\hline
\end{tabular}

Tabla 4. Estado del paciente al mes y al año posterior al trasplante, según ocurrencia de complicaciones médicas y quirúrgicas.

$(17,1 \%), 8(19,1 \%) p=0,00$; con respecto a los que no presentaron este tipo de complicación $1(1,1 \%)$, $3(3,2 \%) p=0,00$ al mes y al año post-TR respectivamente; y comparados también con los que desarroIlaron complicaciones médicas.

También hubo mayor número de fallecidos al mes y al año post-TR en el grupo de pacientes con complicaciones médicas 7 ( $8,1 \%$ ) 0,25, 9 (10,5\%) 0,32 respectivamente, en relación con los que no presentaron este tipo de complicaciones $1(2,0 \%) 0,25$ y $2(4,0$ \%) 0,32 al mes y al año post-TR respectivamente, aunque en este caso las diferencias no resultaron ser estadísticamente significativas.

\section{Discusión}

Las complicaciones médicas infecciosas más frecuentes fueron la infección del tracto urinario y la sepsis respiratoria, lo que se corresponde con los resultados de otras series ${ }^{12,13}$. La infección del tracto urinario constituye la infección más frecuente del trasplante renal que afecta a más del $90 \%$ de los pacientes en las series clásicas y con una incidencia entre 5 y $26 \%$ en las series actuales ${ }^{12}$. Según estudios publicados, la infección del tracto respiratorio inferior, afecta de un 10 a un $40 \%$ de estos pacientes y es en sí misma la causa asociada más frecuente de infección grave y de muerte en el trasplante renal ${ }^{12,13}$.

El paciente que recibe TR presenta una situación de inmunodeficiencia debido a la uremia y al tratamiento dialítico, a ello se añaden otros factores como la pérdida de la integridad de la piel que produce la cirugía, los fármacos inmunosupresores, la infección por determinados virus que amplifican el estado de inmunosupresión, todos estos factores en conjunto hacen que el paciente se encuentre en un estado de gran susceptibilidad a las infecciones ${ }^{12,13}$. En muchos casos las infecciones se corresponden con gérmenes gram negativos, y los factores de riesgo se encuentran en los catéteres vesicales, traumatismo del uréter durante la cirugía y las anomalías asociadas al trasplante renal (como el reflujo vesicoureteral y vejiga neurogénica) sobre todo en pacientes diabéticos, y en la inmunosupresión ${ }^{13}$. 
Las complicaciones médicas no infecciosas más comúnmente halladas fueron la NTA, las crisis hipertensivas, el EAP y las arritmias. En el caso particular de la NTA es la complicación médica más frecuente en la casi absoluta totalidad de las series de pacientes de $\mathrm{TR}^{14-16}$. En nuestro caso, si consideramos que habitualmente trasplantamos con tiempos de isquemia fría superiores a las 20 horas, no es de asombrarse estos resultados, pues es conocido que el tiempo de isquemia es un factor de riesgo muy importante para el desarrollo de NTA ${ }^{13,17,18}$.

Las crisis hipertensivas, EAP y arritmias vienen condicionadas en nuestros pacientes en primer lugar por la aterosclerosis generalizada que suelen presentar los mismos, pero en el período del post-TR temprano aparecen muy comúnmente relacionadas con la administración generosa de líquidos en el transoperatorio y postoperatorio inmediatos, especialmente en aquellos pacientes que presentan NTA y se comportan oligoanúricos en el post-TR. También las drogas pueden favorecer estos episodios, especialmente las altas dosis de esteroides utilizadas en la inducción que favorecen la HTA y las arritmias ${ }^{1}$, que como es lógico pueden influir en la claudicación de cavidades izquierdas y terminar en EAP. Además en nuestros pacientes, que presentan una tasa alta de tratamiento de inducción con terapia cuádruple secuencial que incluye el anticuerpo monoclonal, la aparición de EAP pudiera estar en relación con el uso de esta droga, pues es conocido que el síndrome hipercitoquinémico que esta determina, provoca cambios en las resistencias vasculares pulmonares favoreciendo la aparición de edema alveolar, resultante en EAP 1,19.

Las complicaciones inmunológicas en nuestro estudio muestran un comportamiento similar al de otras series tanto nacionales ${ }^{20}$ como internacionales $^{21}$. Debemos destacar que nuestra frecuencia es menor que la reportada en la bibliografía pues en nuestro estudio nos limitamos a identificar las que se presentaron sólo en la primera semana post-TR. En el análisis de las complicaciones quirúrgicas debemos señalar que en la primera semana post-trasplante las complicaciones vasculares y parenquimatosas son las de mayor importancia, presentándose las trombosis arteriales y venosas, la hemorragia perioperatoria y las roturas renales en nuestra serie con un patrón similar al de otras series pero con una mayor frecuencia absoluta que la observada en otros trabajos. Se destaca como punto de comparación un estudio multicéntrico español realizado en 1992, donde las trombosis arteriales se presentaron en el $2,4 \%$ de los injertos, las trombosis venosas en el $0,9 \%$, el sangramiento en el $2 \%$ y la rotura renal en el $1,5 \%$.

Al evaluar la relación entre supervivencia del injerto al mes y la ocurrencia o no de complicaciones médicas hallamos que la supervivencia era mayor en aquellos sujetos que habían estado exentos de complicaciones médicas. La NTA que fue la complicación médica más frecuente, se ha relacionado de forma casi universal con una pobre supervivencia del injerto ${ }^{13,23}$ incluyéndose dentro de este grupo las denominadas ausencia de función primaria refiriéndose ello a aquellos riñones que una vez injertados nunca tuvieron función, además la presencia de NTA es un factor predisponente a la aparición ulterior de episodios de rechazo que sin lugar a duda lastran la supervivencia de los injertos ${ }^{13,23}$. La toxicidad aguda por CyA es una complicación que suele ser soluble, o sea cuando disminuimos las dosis de CyA se experimenta una recuperación de la función renal pero esta recuperación puede no ser total pues a punto de partida de la toxicidad por CyA se pudiera desarrollar daño irreversible de un grupo de unidades funcionales ${ }^{16,24}$ que no pueden ser rescatadas, especialmente cuando los episodios se suceden en el tiempo.

Las complicaciones médicas también tuvieron una relación significativa con la supervivencia del injerto al año, teniendo un especial significado en este análisis la aparición de episodios de rechazo, los que conducen a una disminución de la masa nefronal y por motivos obvios disminuyen la función renal al año. Además de esto las ITU, especialmente cuando son repetidas determinan la aparición de una nefropatía tubulointersticial que al sumársele el efecto nocivo que tiene la CyA sobre el tubulointersticio renal determina la elevación paulatina de azoados en estos pacientes ${ }^{25}$.

Al analizar las complicaciones quirúrgicas y la supervivencia del injerto al año debe hacerse notar que nos restringimos a aquellas complicaciones derivadas del acto del TR propiamente no incluyéndose las complicaciones derivadas de la extracción del órgano del donante, que sin dudas pueden influir en la supervivencia del injerto, pues cuando se producen accidentes tales como desgarros arteriales 0 venosos 0 
los daños a los uréteres durante la extracción, implican que a la hora de hacer el implante se tenga que realizar una cirugía de banco con reparación del tejido dañado durante la extracción, haciéndose esto a veces difícil y conllevando a que el injerto no se realice en condiciones óptimas.

Todas las complicaciones quirúrgicas sin excepción constituyen verdaderos valladares para la buena evolución de un injerto renal, así en nuestra serie tanto las complicaciones vasculares y parenquimatosas como las urológicas, determinaron una muy pobre evolución del injerto y del paciente tanto al mes como al año del TR. Estos hallazgos lejos de constituir una excepción son una regla en la mayoría de las series revisadas ${ }^{13,20}$, pero debemos destacar que el número de complicaciones quirúrgicas de nuestra serie fue elevado en comparación con la registrada por otros autores ${ }^{22}$.

Existen una serie de elementos que debemos analizar cuando estudiamos la supervivencia del paciente trasplantado, que tienen influencia sobre la misma y que no están en relación con complicaciones médicas o quirúrgicas del post-TR, como por ejemplo la edad del paciente ${ }^{26}$, el origen del órgano ${ }^{17,27,28}$, la presencia de enfermedades sistémicas, como pueden ser la HTA y la DM. La presencia de enfermedades cardiovasculares como condición comórbida en estos sujetos es muy importante pues ellas constituyen la primera causa de muerte entre los pacientes trasplantados ${ }^{13,29}$. Otro elemento que va a marcar de forma decisiva la supervivencia del paciente al año de ser trasplantado es la función renal del injerto pues en la medida que esta sea peor determinará una menor sobrevida del sujeto a punto de partida de todas las condiciones asociadas a la disfunción renal ${ }^{30}$.

Teniendo presente los argumentos anteriores encontramos que la supervivencia fue mejor en aquellos individuos que no presentaron complicaciones médicas, aunque esta diferencia no resultó estadísticamente significativa. Debemos subrayar que nos referimos a las complicaciones médicas propias del trasplante y no a las condiciones comórbidas que pudiera presentar el sujeto con anterioridad, que como antes señalamos influyen de forma notoria sobre la supervivencia del paciente.
Por otra parte, las complicaciones quirúrgicas devenidas del acto del TR si tuvieron una influencia muy deletérea sobre la supervivencia del paciente, téngase presente que muchas de las complicaciones quirúrgicas del post-TR ponen directamente en riesgo la vida del paciente, es este el caso de las trombosis de la arteria renal que puede extenderse al territorio íleo-femoral y comprometer la vida del sujeto; las hemorragias, ya sean por rotura del órgano o por dehiscencia de las suturas vasculares, determinan la aparición de shock hipovolémico de rápida instauración que puede dar al traste con la vida, así mismo las complicaciones urológicas especialmente las fístulas urinarias favorecen la aparición de infecciones que pueden llegar a constituirse en verdaderas septicemias en estos pacientes inmunodeprimidos ${ }^{22}$ y pueden determinar la muerte del sujeto. Sin lugar a dudas, el daño sobre la funcionalidad del injerto que determina la presencia de complicaciones quirúrgicas conllevará a la aparición en este sujeto de las complicaciones de la enfermedad renal crónica provocando una menor supervivencia.

\section{Bibliografía}

1. Trincado Agudo MT, Rodríguez Martínez R, Espinosa Hernández D, Delgado Miranda MI, Bernal Martínez EM, Pérez Campo RG. Atención de enfermería a pacientes con afecciones nefrourológicas. En: Fenton Tait MC, León Román CA. Temas de Enfermería Médico - Quirúrgica. $1^{\text {era }}$ Edición. Ciudad de la Habana: Editorial Ciencias Médicas, 2005: 122-403.

2. Treviño Becerra A. Tratado de Nefrología. México DF: Prado, 2003:1825.

3. Acosta Sariego JR. Bioética desde una perspectiva cubana. Ciudad de la Habana: Centro Félix Varela, 1997: $246-52$.

4. Matesanz R, Miranda B, Fernández M, Felipe $C$, Naya MT. Evolución de la donación y la actividad trasplantadora en España. En: Llach F, Valderrábano F. Insuficiencia Renal Crónica, Diálisis y Trasplante Renal. $2^{\text {da }}$ Edición. Vol 2. Madrid: Ediciones Norma, 1997: 1449-70.

5. Andreu Periz L, Force Sanmartín E. Revisión actualizada sobre el trasplante de órganos: estudio de un caso. Nursing 2005; 23(2):56-65. 
6. Cirera Segura F, Reina Neyra EP, Martín Espejo JL. Diseño de una guía informativa para los pacientes en diálisis susceptibles de ser trasplantados. Rev Soc Esp Enferm Nefrol 2006; 9(1):48 - 53.

7. Ávila Riopedre F, Velazco 0iz Y, Triana del Toro T, Pila Pérez R. Pérdida del injerto renal. Estudio en un trienio. Archivo Médico de Camagüey 2007; 11(1). [Online], [citado 31 de Mayo 2007], Disponible en Internet: http://www.amc.sld.cu/amc/2007/v1lnl2007/2200.htm

8. Manzano Gutiérrez N, Sánchez Castro S. Protocolo de atención de enfermería al receptor de un Trasplante Renal en una unidad de diálisis. Nure Investigación 2006; (24). [Online], [citado 31 de Mayo 2007], Disponible en Internet: http://www.fuden. es/FICHEROS_ADMINISTRADOR/PROTOCOLO/ Nure24_Protocolo.pdf

9. Andreu L, Force E. La enfermería y el trasplante de órganos. $1^{\text {era }}$ Edición. Madrid: Ed Médica Panamericana, 2004.

10. Martín P, Errasti P. Trasplante renal. An Sist Sanit Navar 2006; 29, Suplemento 2:79-92.

11. Montañes P, Sánchez E, Torrubia FJ. Complicaciones quirúrgicas del trasplante renal. Tema monográfico del LVII Congreso Nacional de Urología. Madrid: ENE Ediciones S.A, 1992: 115-132.

12. Bouza $E$, Moreno S. Infecciones después del trasplante renal. En: Llach F, Valderrábano F. Insuficiencia Renal Crónica, Diálisis y Trasplante Renal. $2^{\text {da }}$ Edición. Vol 2. Madrid: Ediciones Norma, 1997: 1624-56.

13. Hernández Fernández M, Ruiz Méndez AS, López Romero J, Ulacia Sánchez J. Evolución del trasplante renal al año. [Online] $7^{\circ}$ Congreso Virtual Hispanoamericano de Anatomía Patológica, 29/01/2006. Disponible en Internet: http://www.conganat. org/7congreso/vistaImpresion.asp?id_trabajo $=236$

14. Rodrigo R, Ruiz J, Piñera C, Fernández-Fresnedo $G$, et al. Creatinine Reduction Ratio on Post-Transplant Day Two as Criterion in Defining Delayed Graft Function. American Journal of Transplantation 2004; 4(7):1163.
15. Borroto Díaz G, Barceló Acosta M, Guerrero Díaz C, Rodríguez Alonso H. Frecuencia y factores de riesgo de la necrosis tubular aguda en el post-trasplante renal inmediato. Revista Médica Cubana 2002; 41(6). [Online], [citado 31 de Mayo 2007], Disponible en Internet: http://bvs.sld.cu/revistas/med/vol41_6_02/ med02602.htm

16. Sarana HD, Mascheroni C. Trasplante Renal. En: Lovesio C. Medicina Intensiva. $5^{\text {ta }}$ Edición. Argentina: Editorial El Ateneo, 2001: 1492-99.

17. Gjertson DW. A multi-factor analysis of kidney graft outcomes at one and five years post-transplantation: 1996 UNOS Update. Clin Transpl. 1996; 343-60.

18. Lechevallier E, Dussol B, Luccioni A, Thirion X, et al. Posttransplantation acute tubular necrosis: risk factors and implications for graft survival. Am J Kidney Dis. 1998 Dec; 32(6):984-91.

19. Vallhonrat $H$, Williams WW, Cosimi $A B$, et al. In vivo generation of $\mathrm{C} 4 \mathrm{~d}, \mathrm{Bb}, \mathrm{iC} 3 \mathrm{~b}$, and SC5b-9 after 0KT3 administration in kidney and lung transplant recipients. Transplantation 1999; 67:253.

20. Cuba de la Cruz M, Guerra Quintana I, Herrera SánchezY, Desdin Sánchez E. Trasplante renal en Holguín. Algunos resultados después de tres años de trabajo. Correo Científico Médico de Holguín 2000; 4(3). [Online], [citado 31 de Mayo 2007] Disponible en Internet: http://www.cocmed.sld.cu/no43/n43ori2.htm

21. Alsina J. El rechazo. Clínica, diagnóstico, histología y tratamiento. En: Llach F, Valderrábano F. Insuficiencia Renal Crónica, Diálisis y Trasplante Renal.

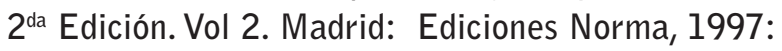
1569-1608.

22. Serrallach N, Franco E, Riera L. Cirugía y complicaciones quirúrgicas del trasplante renal. En: Llach $F$, Valderrábano F. Insuficiencia Renal Crónica, Diálisis y Trasplante Renal. 2da Edición. Vol 2. Madrid: Ediciones Norma, 1997: 1488-1520.

23. Valderrábano F, Alonso Hernández A. Resultados y factores pronósticos en el Trasplante Renal. En: Llach F, Valderrábano F. Insuficiencia Renal Crónica, Diálisis y Trasplante Renal. $2^{\text {da }}$ Edición. Vol 2. Madrid: Editorial Normal, 1997: 1696-1711. 
24. Ubeda Aranda I. Trasplante renal: complicaciones médicas. Clínicas Urológicas de la Complutense, Madrid: Editorial Complutense, 1992 1:113-131.

25. Kamath NS, John GT, Neelakantan N, Kirubakaran $M G$, Jacob CK. Acute graft pyelonephritis following renal transplantation. Transpl Infect Dis 2006; 8(3):140-7. Comment in: Transpl Infect Dis. 2006 Sep; 8(3):125-7.

26. Martín Muñoz M, Villacampa F, Tejido A, Aguirre $E$, et al. El trasplante renal en el paciente de edad geriátrica. Clínicas Urológicas de la Complutense, Servicio de Publicaciones 13CM, Madrid, 1999: 7, 335-350.

27. Johnson SR, Khwaja K, Pavlakis M, Monaco AP, Hanto DW. Older living donors provide excellent quality kidneys: a single center experience (older living donors). Clin Transplant. 2005; 19(5):600-6.
28. Port FK, Dykstra DM, Merion RM, Wolfe RA. Trends and results for organ donation and transplantation in the United States, 2004. Am J Transplant 2005; 5:843.

29. Ostovan MA, Fazelzadeh A, Mehdizadeh AR, Razmkon A, Malek-Hosseini SA. How to decrease cardiovascular mortality in renal transplant recipients. Transplant Proc. 2006 Nov; 38(9):2887-92.

30. Woo $Y M$, Jardine $A G$, Clark $A F$, et al. Early graft function and patient survival following cadaveric renal transplantation. Kidney Int 1999; 55:692. 